\title{
Reliability Analysis-Based Numerical Calculation of Metal Structure of Bridge Crane
}

\author{
Wenjun Meng, ${ }^{1}$ Zhengmao Yang, ${ }^{1}$ Xiaolong Qi, ${ }^{2}$ and Jianghui Cai ${ }^{1}$ \\ ${ }^{1}$ Taiyuan University of Science and Technology, Taiyuan 030024, China \\ ${ }^{2}$ Tongji University, Shanghai 200092, China \\ Correspondence should be addressed to Wenjun Meng; tyustmwj@tyust.edu.cn
}

Received 17 March 2013; Accepted 21 September 2013

Academic Editor: Zhichun Yang

Copyright (c) 2013 Wenjun Meng et al. This is an open access article distributed under the Creative Commons Attribution License, which permits unrestricted use, distribution, and reproduction in any medium, provided the original work is properly cited.

\begin{abstract}
The study introduced a finite element model of DQ75t-28m bridge crane metal structure and made finite element static analysis to obtain the stress response of the dangerous point of metal structure in the most extreme condition. The simulated samples of the random variable and the stress of the dangerous point were successfully obtained through the orthogonal design. Then, we utilized $\mathrm{BP}$ neural network nonlinear mapping function trains to get the explicit expression of stress in response to the random variable. Combined with random perturbation theory and first-order second-moment (FOSM) method, the study analyzed the reliability and its sensitivity of metal structure. In conclusion, we established a novel method for accurately quantitative analysis and design of bridge crane metal structure.
\end{abstract}

\section{Introduction}

Bridge crane metal structure is significant in the bridge crane to host, tract, walk, and brake, which is the most critical part of the performance to determine its safety. As the stability and reliability of metal structure is the guarantee of the entire system, its corresponding analysis, therefore, is crucial before the application $[1,2]$. Currently, there are various uncertainties in the metal structure of bridge crane, such as size parameters, material properties, and external load. These uncertainties have directly influenced the safety and reliability of the metal structure. Therefore, studies using the reliability analysis theory to ensure the stability are encouraging. In addition, due to the different influence degrees of these random parameters, the reliability sensitivity analysis is also essential to obtain dependence of metal structure reliability to each random parameter, which will instruct the optimized design and applicable conditions of the metal structure.

The study introduced a finite element model of bridge crane metal structure and then applied finite element simulation and orthogonal experimental design to obtain the dangerous parts and stress response in the most extreme condition. We utilized BP neural network fitting technology to get an explicit expression of the stress on the design variables.
Besides, random perturbation theory and FOSM method were used for the reliability analysis. The matrix differential technology further contributed to deduce the influence degree of various random parameters on the reliability of the bridge crane metal structure.

\section{Finite Element Analysis of Bridge Crane Metal Structure}

2.1. Finite Element Analysis. ANSYS parametric design language (APDL) was used to establish the finite element model of bridge crane metal structure (Figure 1). Box-type bridge structure model selected shell elements SHELL63 (4-Node Elastic Shell, UX, UY, UZ, ROTX, ROTY, ROTZ) because the unit had capacity to deal with stress stiffening and large deflection [3]. Car track model selected beam element BEAM 189 (3 Node 3-D Quadratic Finite Strain Beam, UX, UY, UZ, ROTX, ROTY, ROTZ), which is based on the Timoshenko beam analysis theory, with default of shear effects and large deformation effects $[3,4]$.

2.2. Stress Response Analysis. We applied finite element analysis software ANSYS to simulate and analyze the metal structure of bridge crane. The stress response in the most severe 


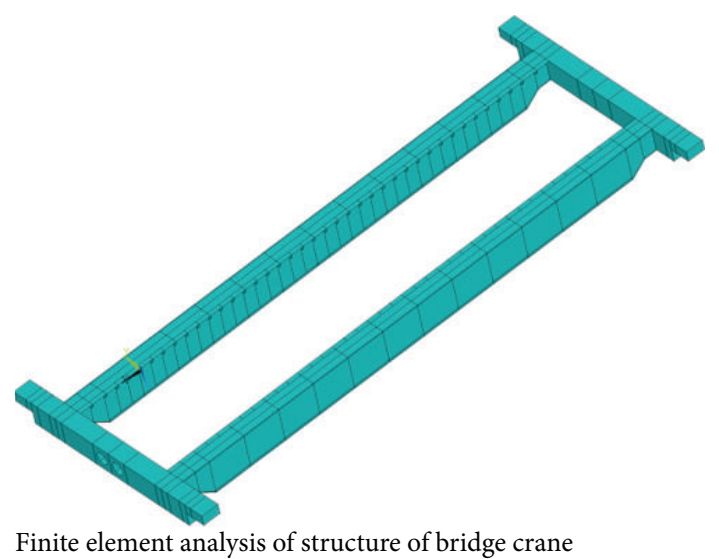

FIGURE 1: Finite element model of bridge crane metal structure.

operating conditions was obtained (Figure 2). With full load, the maximum stress (110 MPa) was observed at the midspan of metal structure and the maximum deformation occurred at the midspan of top flange plate. Meanwhile, the biggest stress existed at the intersection of the lower flange plate, the main web and the diaphragms of box structure (Figure 2). Although the intersection presented the potential fracture and failure, the value did not exceed the material yield strength and conformed to the requirements of static strength and static stiffness. Therefore, it can be concluded that the metal structure will not permanently deform and can meet the needs of safe carrying and running. The intermetal structure von-Mises stress nephogram in the condition of full load was shown in Figure 2.

\section{BP Neural Network Fitting Stress Response}

Since the metal structure of bridge machine is an extremely complex box structure, the function of stress and random design variables is a highly nonlinear and implicit. Therefore, we utilized the orthogonal experimental design method and finite element simulation to get sample data. Then, we combined the BP neural network technology to map the relationship between structure design variables and stress values in dangerous parts, fitting to acquire their explicit expression [5-7].

3.1. Neural Network Training Samples. The study applied orthogonal experimental design method to establish the reasonable quantity and distribution of neural network training samples to accurately express the mapping relationship of neural network model. According to the reliability theory, we selected the simulated full-load condition as the object and obtained the data of random variable as well as the stress response (Table 1 ).

The output samples of stress response of dangerous parts could be gained through the experimental design. It would be further used as the training samples of BP neural network to eventually fit required explicit expressions: $S=$ $\left(W, H, B, E, e_{-} Y Y B, e_{-} F B\right)$.

Training samples of the neural network model was obtained by using the orthogonal table $L_{25}\left(5^{6}\right)$; that is,

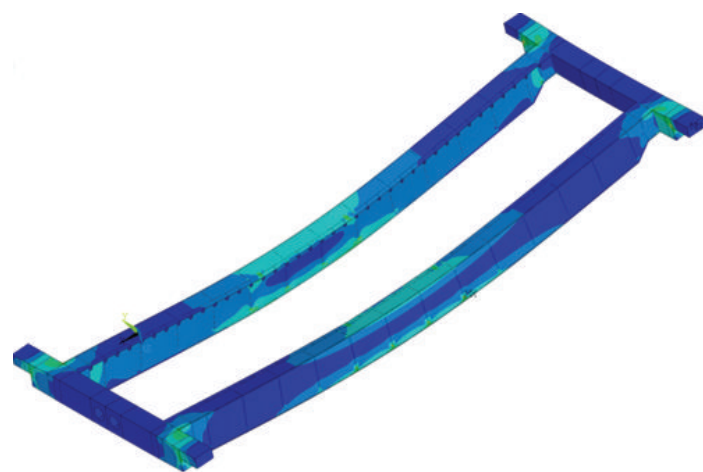

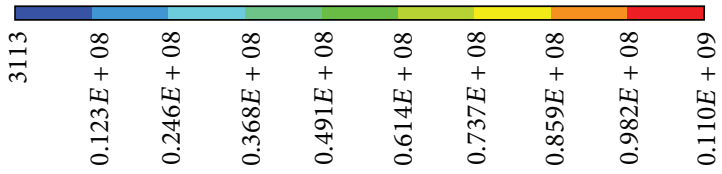

Finite element analysis of structure of bridge crane

FIgURE 2: Stress nephogram of metal structure under full-load condition.

TABLE 1: Random variable and its statistical properties of bridge crane metal structure.

\begin{tabular}{lccc}
\hline $\begin{array}{l}\text { Random variable } \\
\text { (factors) }\end{array}$ & Meaning & Average & $\begin{array}{c}\text { Standard } \\
\text { deviation }\end{array}$ \\
\hline$W / 10^{6} \mathrm{~N}$ & Lifting load & 250 & 20 \\
$H / \mathrm{mm}$ & Main beam height & 8155 & 366 \\
$B / \mathrm{mm}$ & Main beam width & 4108 & 207 \\
$E / \mathrm{MPa}$ & Elastic modulus & 206 & 10 \\
$e_{-} Y Y B / \mathrm{mm}$ & Upper and lower flange & 14 & 4.2 \\
$e_{-} F B / \mathrm{mm}$ & plate thickness & 12 & 2.5 \\
\hline
\end{tabular}

TABLE 2: Orthogonal factors and the level.

\begin{tabular}{lcccccc}
\hline Level & $W$ & $H$ & $B$ & $E$ & $e_{-} Y Y B$ & $e_{-} F B$ \\
\hline 1 & 230 & 8000 & 3800 & 196 & 11 & 6 \\
2 & 240 & 8100 & 3900 & 201 & 12 & 7 \\
3 & 250 & 8200 & 4000 & 206 & 13 & 8 \\
4 & 260 & 8300 & 4100 & 211 & 14 & 9 \\
5 & 270 & 8400 & 4200 & 216 & 15 & 10 \\
\hline
\end{tabular}

the total number of samples was 25 , the factors were 6 , each factor had 5 levels [4] (Table 2). The stress response of its corresponding 25 samples, as output variables of neural network model, was calculated by the finite element model.

3.2. Neural Network Model. For the 6 random variables of bridge crane metal structure system, BP neural network input layer had 6 neurons and the hidden layer had 15 neurons. Each neuron of the output layer was described as the stress response of the bridge crane metal structure.

To get the stress response neural network model of the global significance, each random variable in its feasible region took 5 discrete values (horizontal) and generated 25 design samples as the training set by the combination of 
orthogonal test. Then, finite element simulation experiments were carried out on all samples by ANSYS software.

3.3. Neural Network Learning. After the model structure of BP neural network was determined, the toolbox (NNET Toolbox) of MATLB was used in training the network using the input sample set and the output sample set to achieve a given input-output mapping relationship and further correct the thresholds and weights of the network [8].

Some neurons reached saturation due to the large difference in the magnitude of each variable in the original sample. As a result, the input samples should be normalized first and then selected the neurons pass for hidden layer and output layer as $\tan \operatorname{sig}()$ and purelin(), respectively [9]. Meanwhile, the training function trainlm in LM (Levenberg-Marquardt) algorithm was selected for training network when convergence speed and accuracy were taken into consideration.

3.4. Neural Network Generalization Test. Typically, the training patterns included in the training set are only part of the source data set. Even if the network was trained by all the patterns within the training set, it also could not guarantee that the test by another mode could give satisfactory results. Therefore, the generalization ability of fitting functions of 25 new samples, as a test set, is still needed to be examined after the network learning. From the result of test error (Figure 3), the fitted model met the requirement of accuracy and could replace the finite element simulation for reliability analysis.

In addition, the convergence of the neural network training was demonstrated (Figure 4).

\section{Reliability Analysis of Bridge Crane Metal Structure}

A set of basic random variables $X=\left(X_{1}, X_{2}, \ldots, X_{n}\right)^{T}$ features the design dimensions, the material properties, the load that are normally distributed. Its joint probability density function is $f_{X}(X)$. The limit state function of the bridge crane metal structure is expressed as

$$
g(X)=g\left(X_{1}, X_{2}, \ldots, X_{n}\right) .
$$

$g(X)<0$ represents the failure state, $g(X)=0$ is limit state, and $g(X)>0$ is the security state. Based on the probability theory, the reliability is calculated using multiple integrals in reliable domain when $g(X)>0$; namely,

$$
R=\int_{g(X)>0} \cdots \int I[g(x)] f\left(x_{1}, x_{2}, \ldots, x_{n}\right) d x_{1} d x_{2} \cdots d x_{n},
$$

when $g(X)>0, I[g(X)>0]=1$; when $g(X)<0, I[g(X)<$ $0]=0$.

Based on the perturbation theory, $X$ and $g(X)$ can be expanded as follows:

$$
\begin{gathered}
X=X_{d}+\Delta X_{p}, \\
g(X)=g_{d}(X)+\Delta g_{p}(X),
\end{gathered}
$$

where subscript $d$ and $p$ separately denote the determining part and random part of random variable and have zero

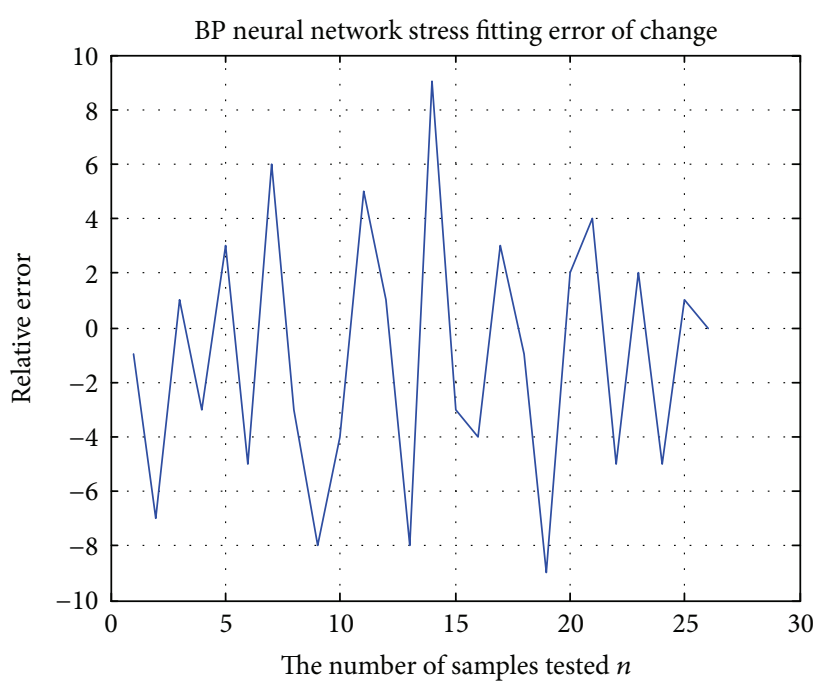

Figure 3: Relative error of BP neural network fitted model.

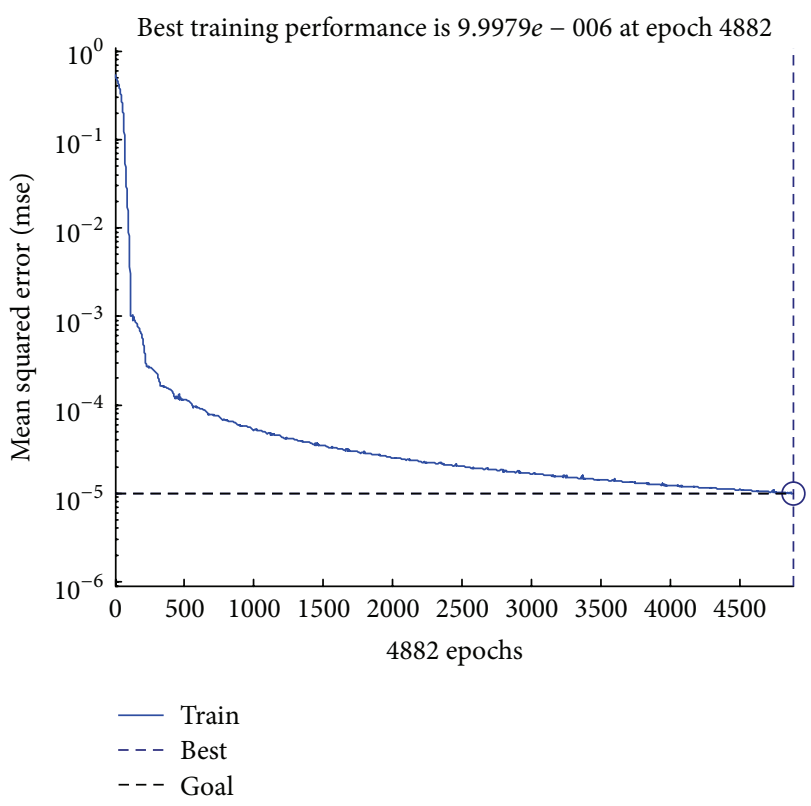

FIGURE 4: Grid training convergence curve.

mean; $\Delta$ denotes higher-order infinitesimal. Based on the random analysis theory, the mean and variance of formula (3) are as follows:

$$
\begin{gathered}
\mu_{g}=E[g(X)]=E\left[g_{d}(X)\right]+\Delta E\left[g_{p}(X)\right]=g_{d}(X) \\
\sigma_{g}^{2}=\operatorname{Var}[g(X)]=\Delta^{2} E\left[\left(\frac{\partial g_{d}(X)}{\partial X^{T}}\right) \Delta X_{p}\right] \\
=\left(\frac{\partial g_{d}(X)}{\partial X^{T}}\right) \operatorname{Var}(X) .
\end{gathered}
$$

Based on the FOSM method, take a linear term of the Taylor expansion of limit state equation and calculate the probability of the function greater than zero by basic random variables of 
first moment (mean) and second moment (variance). Reliability is expressed as $R$ :

$$
R=\Phi(\beta)=\Phi\left(\frac{\mu_{g}}{\sigma_{g}}\right)=\Phi\left(\frac{E[g(X)]}{\sqrt{\operatorname{Var}[g(X)]}}\right)
$$

Set $\sigma$ for the corresponding stress intensity of the dangerous point of bridge machine metal structure. Then, the limit state function is written as

$$
g(X)=\sigma-S\left(W, H, B, E, e_{-} Y Y B, e_{-} F B\right)
$$

\section{Reliability Sensitivity Analysis of Bridge Crane Metal Structure}

Based on the FOSM method and matrix differential, the mean and variance sensitivity of the metal structure reliability to each random variable are expressed as follows:

$$
\begin{gathered}
\frac{d R_{i}}{d \bar{X}^{T}}=\frac{\partial R_{i}}{\partial \beta_{i}} \frac{\partial \beta_{i}}{\partial \mu_{g_{i}}} \frac{\partial \mu_{g_{i}}}{\partial \bar{X}^{T}}, \quad(i=1,2, \ldots, n), \\
\frac{d R_{i}}{d \operatorname{Var} X}=\frac{\partial R_{i}}{\partial \beta_{i}} \frac{\partial \beta_{i}}{\partial \sigma_{g_{i}}} \frac{\partial \sigma_{g_{i}}}{\partial \operatorname{Var} X}, \quad(i=1,2, \ldots, n),
\end{gathered}
$$

where

$$
\begin{gathered}
\frac{\partial R_{i}}{\partial \beta_{i}}=\varphi\left(\beta_{i}\right), \quad \frac{\partial \beta_{i}}{\partial \mu_{g_{i}}}=\frac{1}{\sigma_{g_{i}}}, \\
\frac{\partial \mu_{g_{i}}}{\partial \bar{X}^{T}}=\left[\frac{\partial \bar{g}_{i}}{\partial X_{1}} \frac{\partial \bar{g}_{i}}{\partial X_{2}} \cdots \frac{\partial \bar{g}_{i}}{\partial X_{n}}\right], \\
\frac{\partial \beta_{i}}{\partial \sigma_{g_{i}}}=-\frac{\mu_{g_{i}}}{\sigma_{g_{i}}^{2}}, \\
\frac{\partial \sigma_{g_{i}}}{\partial \operatorname{Var} X}=\frac{1}{2 \sigma_{g_{i}}}\left[\frac{\partial \bar{g}_{i}}{\partial X} \otimes \frac{\partial \bar{g}_{i}}{\partial X}\right] .
\end{gathered}
$$

\section{Results and Analysis}

According to formula (5), the reliability of the metal structure is $R=0.999866$. Use Monte Carlo simulation for test to calculate 106 times. The reliability is $R_{\mathrm{MCS}}=0.999764$. Relative error is as follows:

$$
\varepsilon_{R}=\left|\frac{R-R_{\mathrm{MSC}}}{R_{\mathrm{MCS}}}\right|=1.306 \times 10^{-4} .
$$

Based on the known conditions and reliability calculation results, the reliability sensitivity of random variables including $d R_{i} / d X^{T}$ and $d R_{i} / d \operatorname{Var} X$ can be calculated according to formula (7) [10]. The mean sensitivity of the bridge machine metal structure is as follows:

$$
\frac{d R}{d X^{T}}=\left[\begin{array}{c}
R(W) \\
R(H) \\
R(B) \\
R(E) \\
R\left(e_{-} Y Y B\right) \\
R\left(e_{-} F B\right)
\end{array}\right]=\left[\begin{array}{c}
4.043 \times 10^{-3} \\
1.692 \times 10^{-4} \\
1.432 \times 10^{-4} \\
3.453 \times 10^{-5} \\
3.308 \times 10^{-7} \\
4.682 \times 10^{-8}
\end{array}\right] .
$$

Variance sensitivity is as follows:

$$
\frac{d R}{d \operatorname{Var} X}=\left[\begin{array}{c}
R_{\operatorname{Var}}(W) \\
R_{\operatorname{Var}}(H) \\
R_{\operatorname{Var}}(B) \\
R_{\operatorname{Var}}(E) \\
R_{\operatorname{Var}}\left(e_{-} Y Y B\right) \\
R_{\operatorname{Var}}\left(e_{-} F B\right)
\end{array}\right]=\left[\begin{array}{c}
-6.385 \times 10^{-10} \\
-4.902 \times 10^{-11} \\
-7.746 \times 10^{-13} \\
-8.658 \times 10^{-11} \\
-3.537 \times 10^{-7} \\
-6.135 \times 10^{-9}
\end{array}\right] .
$$

It can be drawn from the sensitivity matrix of $d R_{i} / d X^{T}$ that the reliability sensitivity of bridge crane metal structure can be influenced by lifting load $W$, main beam height $H$, main beam width $B$, and elastic modulus $E[10]$. Moreover, the influence degree of 6 variables on structural reliability in decreasing order are lifting load $W$, main beam height $H$, main beam width $B$, elastic modulus $E$, flange plate thickness $e_{-} Y Y B$, and web thickness $e_{-} F B$.

\section{Conclusions}

(i) The study applied the neural network technology and orthogonal experimental design method to solve the reliability calculation with the implicit limit state function and to obtain the explicit expression of the random variable and the stress response of bridge crane metal structure.

(ii) The integrated use of orthogonal experimental design method and finite element simulation test in the reliability analysis engineering can significantly reduce the design costs and markedly shorten the cycle.

(iii) Based on the theory of reliability design and sensitivity, we made a derivation analysis of the reliability and its sensitivity and then established a basis for accurately quantitative analysis of the reliability of bridge crane metal structure.

\section{Acknowledgments}

The project is supported by the Natural Science Foundation Committee (51075289); National Natural Science Foundation International Cooperation and Exchanges Project (51110105011); 2012 Higher School Specialized Research Fund for the Doctoral Program Joint Funding Issues (20121415110004); Research Foundation for Returning Scholar in 2009 of Shanxi province (20091074); International Scientific and Technological Cooperation Projects in 2010 of Shanxi province (2010081039); Shanxi Natural Science Foundation (2011011019-3); 2010 Scientific Star Project in Taiyuan 
city (2010011605); 2009 Shanxi province Graduates' Excellent and Innovative Project (20093099); Shanxi province 2011 UIT Item, TYUST 2010 UIT Item (201008X); Doctor Start up Item and Characteristics \& Leading Academic Discipline Project of Universities of Shanxi Province.

\section{References}

[1] D. N. Thatoi, H. C. Das, and D. R. Parhi, "Review of techniques for fault diagnosis in damaged structure and engineering system," Advances in Mechanical Engineering, vol. 2012, Article ID 327569, 11 pages, 2012.

[2] N. Saravanan, V. N. S. K. Siddabattuni, and K. I. Ramachandran, "Fault diagnosis of spur bevel gear box using artificial neural network (ANN), and proximal support vector machine (PSVM)," Applied Soft Computing Journal, vol. 10, no. 1, pp. 344360, 2010.

[3] P. Zeng, L. Lei, and G. Fang, Finite Element Analysis Guide: Modeling and Analysis of Structure, China Machine Press, Beijing, China, 2013 (Chinese).

[4] X. He and Y. Zhang, "Reliability sensitivity design of mechanical systems," Machinery Manufacturing, vol. 5, pp. 26-28, 2009 (Chinese).

[5] M. Hohenbichler and R. Rackwitz, "Sensitivity and importance measures in structural reliability," Civil Engineering Systems, vol. 3, no. 4, pp. 203-209, 1986.

[6] K. Xu, M. Xie, L. C. Tang, and S. L. Ho, "Application of neural networks in forecasting engine systems reliability," Applied Soft Computing Journal, vol. 2, no. 4, pp. 255-268, 2003.

[7] T. Chen, H. Chen, and R.-W. Liu, "Approximation capability in $\mathrm{C}(\mathrm{Rn})$ by multilayer feedforward networks and related problems," IEEE Transactions on Neural Networks, vol. 6, no. 1, pp. 25-30, 1995 (Chinese).

[8] K. Zhou and Y. Kang, The Neural Network Model and Its MATLAB Simulation Program Designed, Tsinghua University Press, Beijing, China, 2005.

[9] G. I. Schuëller, "On the treatment of uncertainties in structural mechanics and analysis," Computational Stochastic Mechanics, vol. 85, no. 5-6, pp. 235-243, 2007.

[10] H. Xiangdong, Z. Yimin, and W. Bangchun, "Reliability sensitivity design of mechanical systems," Machinery Manufacturing, vol. 47, no. 5, pp. 26-28, 2009. 


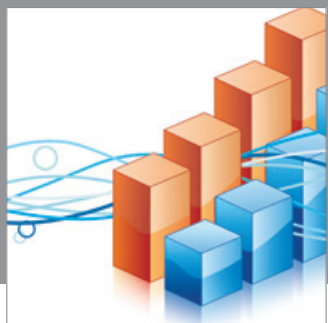

Advances in

Operations Research

mansans

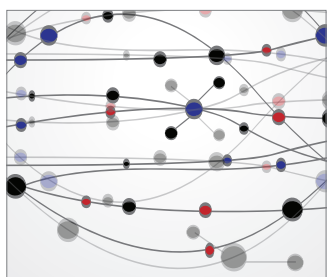

The Scientific World Journal
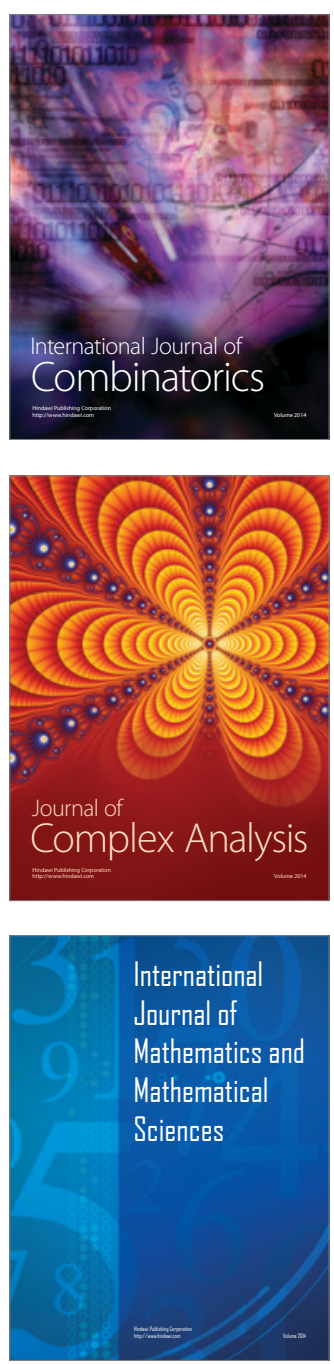
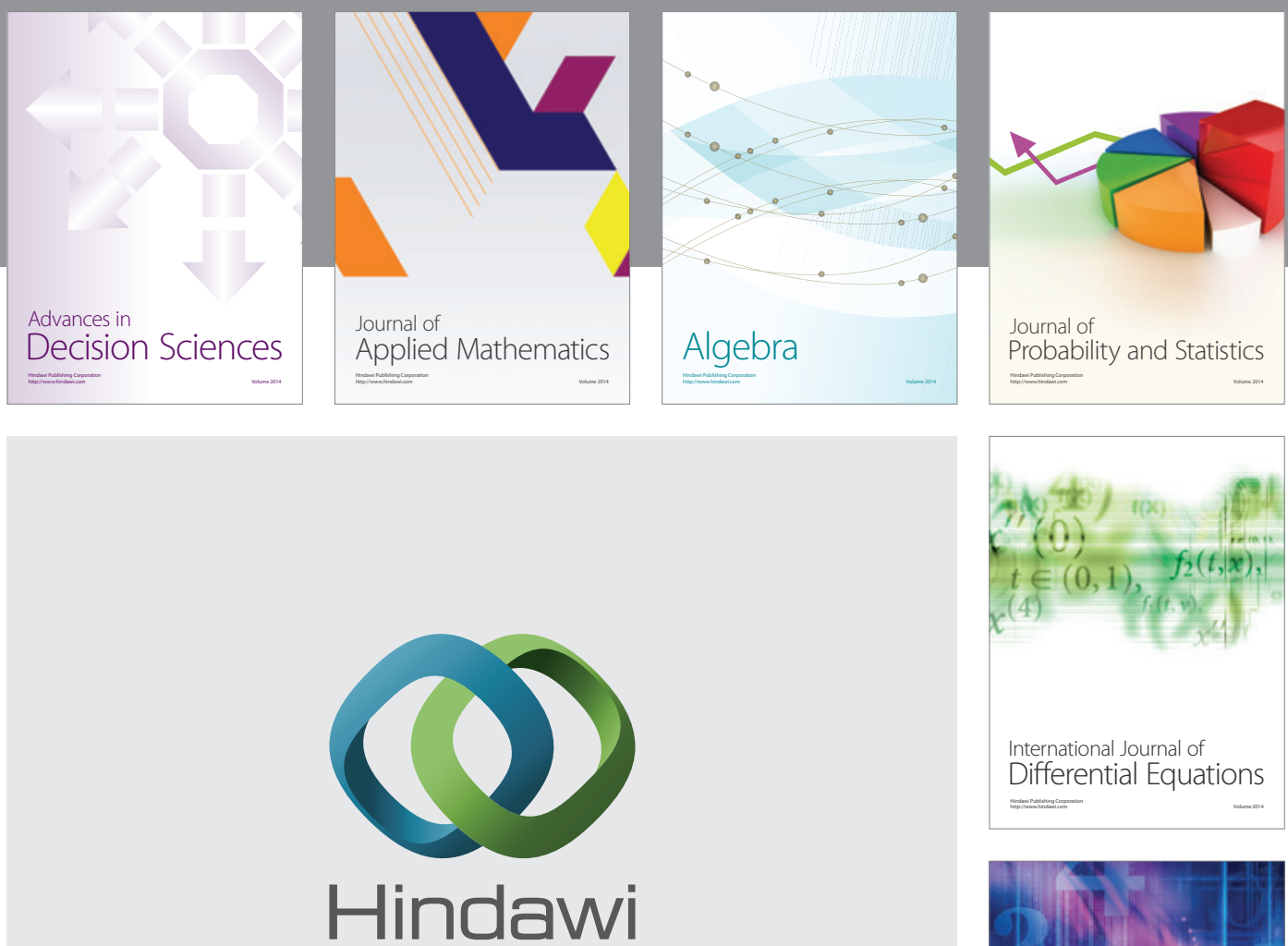

Submit your manuscripts at http://www.hindawi.com
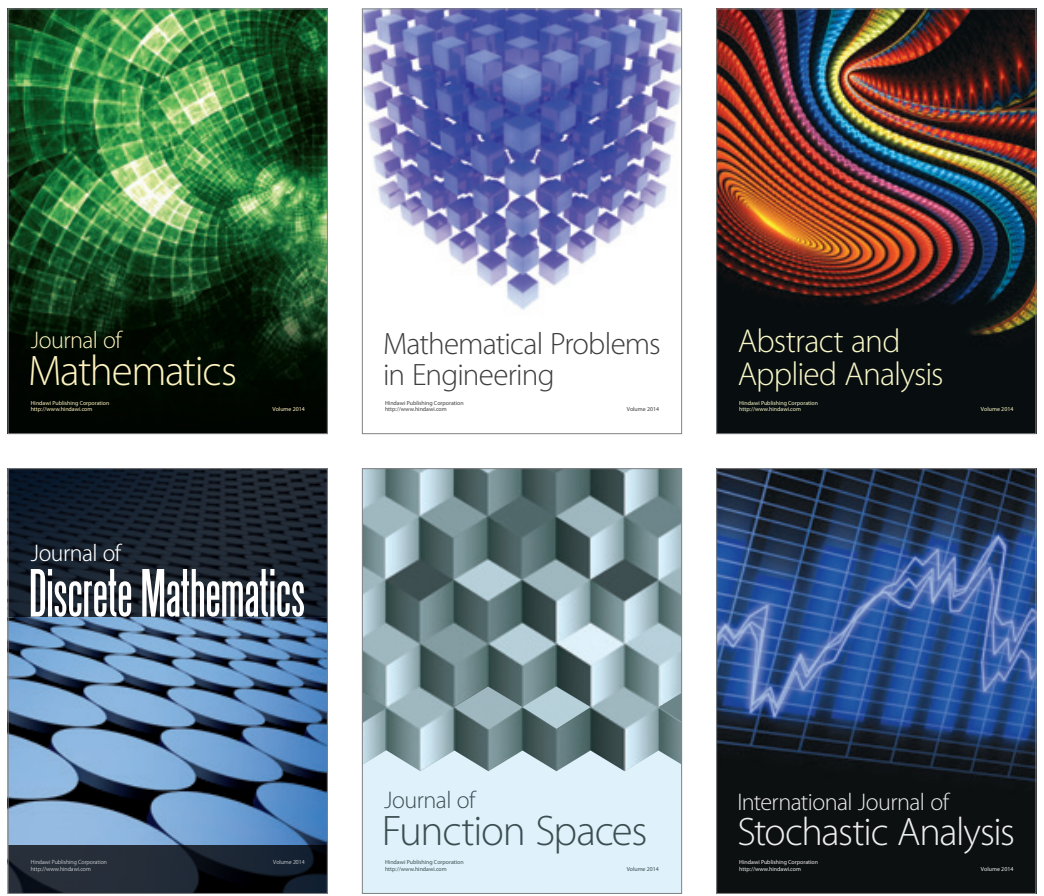

Journal of

Function Spaces

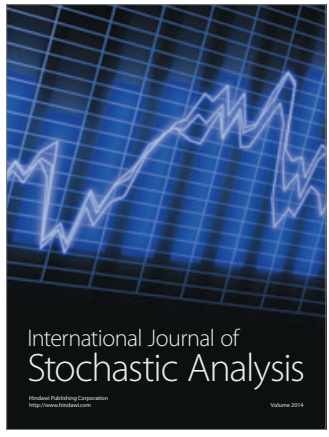

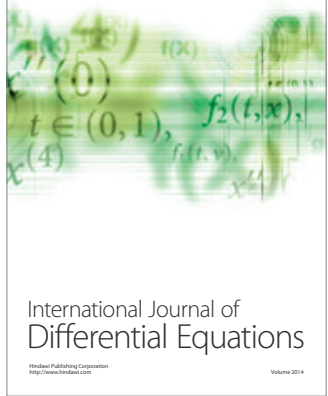
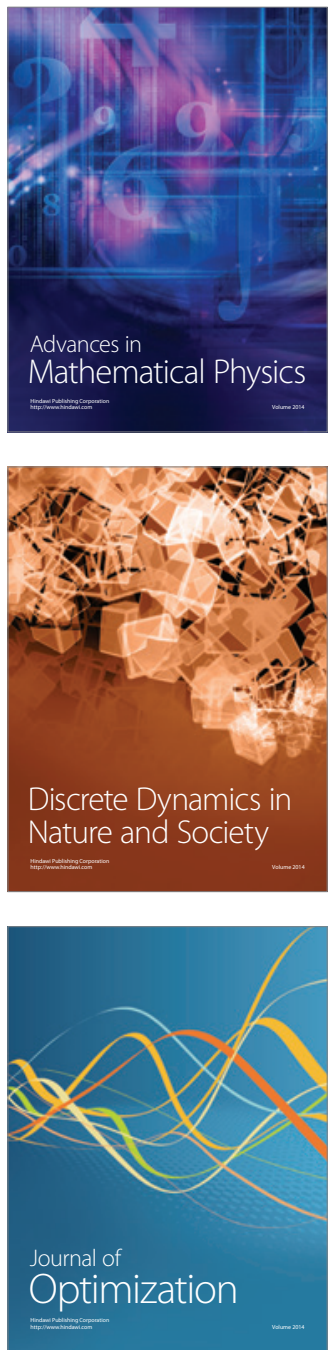